\title{
The Workflow Modeling Research Based on the Expanded P/T System
}

\author{
Long-da Huang \\ Power Automation Department \\ China Electric Power Research Institute \\ Nanjing, China \\ huanglongda@epri.sgcc.com.cn
}

Jin Liu

Jincheng College

Nanjing University of Aeronautics and Astronautics

Nanjing, China

liujin_jc@nuaa.edu.cn

\author{
Jun-song Wang \\ Institute of Computing \& Software \\ Nanjing College of Information Technology \\ Nanjing, China \\ wangjs@njcit.cn \\ Su-yan Long \\ Power Automation Department \\ China Electric Power Research Institute \\ Nanjing, China \\ longsuyan@epri.sgec.com.cn
}

\begin{abstract}
A workflow modeling method based on the expanded $\mathrm{P} / \mathrm{T}$ system (hereafter to be called the $\mathrm{xP} / \mathrm{T}$ system) is put forward to make the correctness verification of the graphical modeling easier and simplify the complication of Petri net modeling. Meanwhile the correctness verification of the modeling is given. The modeling method makes it simpler in the aspects of graphical indication and program implementation by allowing the value of the weight function to be zero and taking the transition as the 'OR' node to implement conditional and iterative routing. In addition it preserves the advantage that Petri net modeling owns.
\end{abstract}

Keywords- workflow modeling; expanded P/T system; routing structure; workflow model correctness;

\section{INTRODUCTION}

Workflow modeling provides for a formal mathematics model supporting process definition, process analysis and process evolution. The core of workflow modeling is the definition of the process, including the activities of the process and the sequence relationships between activities [1-2]. Current workflow models can be divided into two categories: one is the intuitive graphical language or text language to define workflow processes. This method is mainly a user-oriented definition, which is convenient for users, also conducive to the realization of the actual system. Meanwhile the essential characteristics cannot be described, let alone the process analysis and evaluation. The other is the model with the formal and mathematical description, such as Petri net [3-5].

Petri net is a precisely defined mathematical model [67]. It has the strict formalization definition and an intuitive graphical representation [8]. Moreover, Petri net has the rich system-describing methods and system behavior analysis techniques [9], but also provide a solid concept foundation for the computer science. Petri net makes it possible to establish the mathematical model for describing the system performance [10], and it provides a powerful tool for describing the concurrent, asynchronous, distributed and stochastic characteristics of complex systems. However, because most of the existing theories are based on the advanced modeling of Petri net [11-12], the program implementation is very complicated, which greatly limits the extension in the enterprise applications.
The workflow property data are introduced into $\mathrm{P} / \mathrm{T}$ system; meanwhile the weight function of the $\mathrm{P} / \mathrm{T}$ system can take a value of zero. In the above two foundations the $\mathrm{xP} / \mathrm{T}$ system is proposed, which makes workflow modeling easier and maintains the advantages of Petri net modeling.

1 The $\mathrm{xP} / \mathrm{T}$ System Definition

Definition 1: $\mathrm{xP} / \mathrm{T}$ System

An $\mathrm{xP} / \mathrm{T}$ system is a quintuple $\Sigma=(\mathrm{S}, \mathrm{T}, \mathrm{F}, \mathrm{Wx}, \mathrm{M} 0)$, which satisfies the following conditions:

i) $\mathrm{N}=(\mathrm{S}, \mathrm{T}, \mathrm{F})$ is a directed net, which is called the base net of $\Sigma$. $\mathrm{N}$ has one source place $\mathrm{i}(\mathrm{i} \in \mathrm{S})$ and one sink place $\mathrm{o}(\mathrm{o} \in \mathrm{S})$, which respectively meets $* \mathrm{i}=$ and $\mathrm{o}^{*}=$;

ii) $\mathrm{Wx}$ and M0 are respectively the extended weight function and initial marking. Wx is an expression function consisting of workflow property variables, and M0 is called initial marking.

After the initial marking is defined, the scheduling mechanism of the entire workflow model will be implemented under the control of the transition conditions and consequences.

\section{WORKFLOW MODELING BASED ON THE $\mathrm{XP} / \mathrm{T}$ SYSTEM}

The parallel routing is implemented with the transition as the AND-split, and the conditional and iterative routings are implemented with the place as the OR-split in the traditional Petri nets. The $\mathrm{xP} / \mathrm{T}$ system modeling is uniformly implemented with the transition as the ANDsplit and OR-split, which makes the conditional routing and iterative routing modeling more concise, clear, and makes the programs of the unified modeling theory convenient to be implemented. The weight function value of traditional $\mathrm{P} / \mathrm{T}$ system is non-zero. Meanwhile, in order to dynamically describe the transition of the workflow instance and make it flexible to dynamically interact between the workflow engine and the higher-level application program, the workflow property data are directly introduced into $\mathrm{P} / \mathrm{T}$ system weight function expression, and weight function value can be set zero, on the base of which, $\mathrm{xP} / \mathrm{T}$ system is put forward. The expression value of the $\mathrm{xP} / \mathrm{T}$ system weight function can dynamically change with the change of workflow property data, so the transition of workflow instance can be 
controlled. At the same time, the capacity function is not limited, which means that the space of the resources is large enough, and collision will not happen. In addition, the implementing program becomes simpler.

\section{A. The routing structure for the $x P / T$ system workflow model}

1The following four routings of the workflow model based on the $\mathrm{xP} / \mathrm{T}$ system will be introduced, i.e., sequential routing, parallel routing (AND-split and ANDjoin), conditional routing (OR-split and OR-join) and iterative routing. The $\mathrm{xP} / \mathrm{T}$ system improves the conditional routing and iterative routing, but the sequential routing and the parallel routing remains the same with the $\mathrm{P} / \mathrm{T}$ system. (Notes: weight function value must be 0 or 1 and 1 can be omitted.)

i) Sequential routing (Figure. 1): The routing is simplest route, and it means sequentially perform two tasks, and dependency of the tasks is clear.

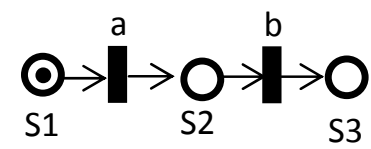

Figure 1. Sequential Routing of P/T System and xP/T System

ii) Parallel routing (Figure. 2): The routing represents two or more tasks in different splits can be performed simultaneously in any order, also known as concurrent routing. In this case, the tasks in different splits have not direct dependencies. The concurrent tasks start with the AND-split, and end with the AND-join. As shown, when task a is triggered, the two output places S2 and S3 , respectively increase by one token, thereby making task $b$ and $\mathrm{c}$ are enabled, then $\mathrm{b}$ and $\mathrm{c}$ may be execute concurrently, because there is no dependencies between them. When task $\mathrm{b}$ and $\mathrm{c}$ are completed, the corresponding output place S4 and S5 have increased by one token, which makes task $\mathrm{d}$ can be executed. The condition that task ' $\mathrm{d}$ ' can be performed is the condition of the transition firing in the $\mathrm{xP} / \mathrm{T}$ system, which requires each input place of ' $\mathrm{d}$ ' meet the condition $\mathrm{M}(\mathrm{s}) \geqslant \mathrm{Wx}(\mathrm{s}, \mathrm{d})$. It can be seen that the AND-join plays a role synchronizing all tasks of the AND-split.

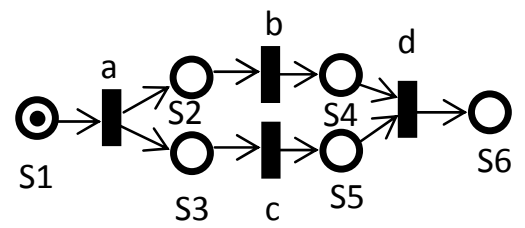

Figure 2. Parallel Routing of $\mathrm{P} / \mathrm{T}$ System and $\mathrm{xP} / \mathrm{T}$ System

iii) Conditional routing (Figure. 3, Figure. 4): when a task is completed someone can be executed in the subsequent tasks. Different transitions may be enabled by sharing input space in the $\mathrm{P} / \mathrm{T}$ system, as shown in Figure. 3. When transition $a$ occurs, the token in the input place $S 1$ will be consumed. In the meantime, the output places, i.e., $S 2, S 3$ and $S 4$ will respectively increase by one token, thereby causing both transition $b$ and $c$ fire, but only a transition occurs because after the occurrence of either $b$ or $c$, the token in the place $S 2$ will be consumed, resulting in that another no longer occurs. So the conditional routing of the transition $b$ and $c$ is realized. In the $\mathrm{xP} / \mathrm{T}$ system conditional routing is controlled by the Boolean value of logical expression consisting of workflow case variable. The logical expression meets javascript syntax, and it is implemented by an open source components apache BSF, and realizes that the logical mapping from the logical expression to the numeric expression, i.e., the logical 'true' is mapped to 1 and the logical 'false' is mapped to 0 . When you need to choose a successor to perform from the $\mathrm{N}$ tasks, the maximum of variables you need to define is $\left\lceil\log _{2} \mathrm{~N}\right\rceil\left(\log _{2} \mathrm{~N}\right\rceil$ means the maximum integer which is not more than $\log _{2} \mathrm{~N}$ ). In Figure. 4, for example, if there two splits of task $a$, one workflow property variable $t$ can meet the need (assuming that the current value of workflow case variable $t$ is true). The two splits is expressed as $t$ and !t i.e., the weight function value of the corresponding output arc is 0 and 1 . After task $a$ is finished, place $S 2$ increase by one token, but place $S 3$ does not increase the token, that is, the token number of $S 3$ is still 0 . At this time task $b$ is enabled, while task $c$ cannot fire. After task $b$ is executed, the output place of $b, S 4$ increase by one token. At this time the weight of the output arc of $S 4$ is $t$, i.e., 1, and the token number of place $S 5$ is 0 . Because the input place ' $\mathrm{S} 4$ ' satisfies $M(s 4)=1>=W(s 4, d)=t=1$, and $M(s 5)=$ $0>=W(s 4, d)=! t=0$, i.e., the transition $d$ is enabled according the $\mathrm{xP} / \mathrm{T}$ system trigger conditions. Thus, as long as the task in any one OR-split is finished, the corresponding OR-join transition can fire. Special attention should be paid that conditional routing must begin with OR-split, and join with OR-join.

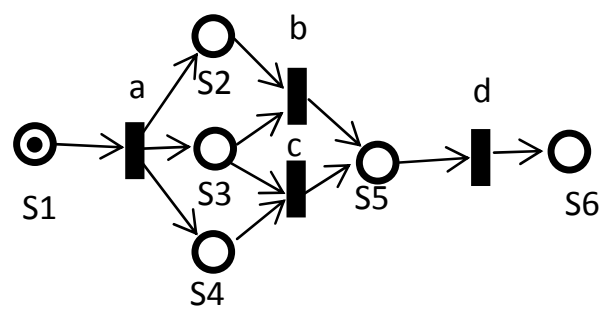

Figure 3. Conditional Routing of $\mathrm{P} / \mathrm{T}$ System

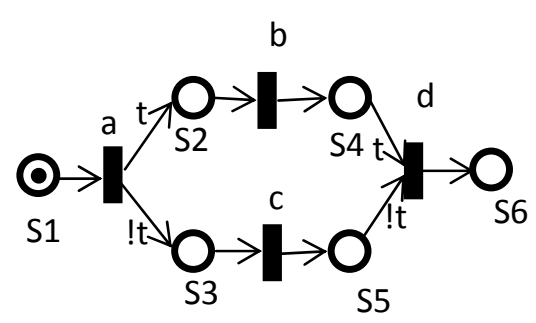

Figure 4. Conditional Routing of $\mathrm{xP} / \mathrm{T}$ System 
iv) Iterative routing (Figure. 5, Figure. 6): In the general case, the execution frequency of each task does not exceed once in a workflow case, but sometimes it may be necessary for a particular task to be executed several times. For example, when a task needs to be repeated until the testing conditions are met. In fact, the iterative routing is a special conditional routing.

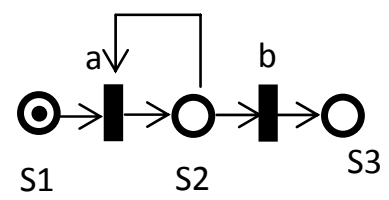

Figure 5. Iterative Routing of P/T System

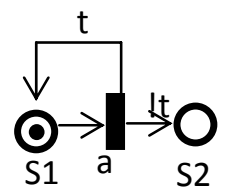

Figure 6. Iterative Routing of $\mathrm{xP} / \mathrm{T}$ System

\section{B. The correctness verification of the workflow model}

To verify the correctness of workflow model based on Petri nets, the following aspects mainly are considered, that is the reachability, the liveness, the integrity and the boundedness :

i) Reachability: when analyzing Petri nets, the following methods can be used, such as reachability tree or reachability diagram, matrix equation, reduction or decomposition. The weight function of the $\mathrm{xP} / \mathrm{T}$ system is a logical expression of a workflow case variables, so matrix is more reasonable to prove its reachability.

ii) Liveness: For any marking in the reachable markings set of the initial marking 'M0', if there is always a transition sequence which makes the transition ' $t$ ' fire , the transition ' $\mathrm{t}$ ' is called alive.. If all the transitions of the $\mathrm{xP} / \mathrm{T}$ systems are alive, the system is called alive.

iii) Integrity: The Petri net with the integrity is reachable completely, which can describe all the circumstances that may arise in the transaction processing system.

vi) Boundedness: The workflow modeling based the $\mathrm{xP} / \mathrm{T}$ system is just to achieve control process, the concept of resources is reflected by the built-in resource expression, so the boundedness is not important in the $\mathrm{xP} / \mathrm{T}$ system.

1) Reachability verification

Definition 2 (Place single step dependency)

For $\forall S i, S j \in S$, if $\mathrm{Si}^{*} \cap * \mathrm{Sj} \neq \phi$, then there exists single step dependency between the place $S i$ and the place $\mathrm{Sj}$, written $\mathrm{Si} \rightarrow \mathrm{Sj}$.

Definition 3 (Single step dependency matrix)

$\mathrm{N}$ order matrix $\mathrm{C}$ is defined as follows: $\mathrm{C}(\mathrm{i}, \mathrm{j})=1$, if $S i \rightarrow S j$, otherwise $\mathrm{C}(\mathrm{i}, \mathrm{j})=0$, then matrix $\mathrm{C}$ is called single step dependency matrix.
Definition 4 (Place Dependency)

э $\mathrm{Si}, \mathrm{Sk} \in \mathrm{S}, \mathrm{Si} \rightarrow \mathrm{Sk}$ Denotes $\mathrm{Sk}$ depends $\mathrm{Si}$, which must satisfy one of the following conditions:

$S i \rightarrow S k$;

э $S j \in S$, meets $S i \rightarrow S j, S j \rightarrow S k$.

Definition 5 (Place Dependency Set)

If $\mathrm{Di}=\{\mathrm{Sj} \mid \mathrm{Sj} \rightarrow \mathrm{Si}\}, \mathrm{Di}(1 \leq i \leq n)$ is the place dependency set of Si.

Algorithm 1 reachability verification algorithm of the $\mathrm{xP} / \mathrm{T}$ system:

i) Clear the dependency sets of all the places: For each $\mathrm{Si}$ in $\mathrm{S}$

$$
\text { Di }=\phi \text {; }
$$

ii) tempC $=\mathrm{C}$;

$$
\text { for }(\mathrm{k}=1 ; \mathrm{k}<=\mathrm{n} ; \mathrm{k}++)
$$

Iterating through all the

$$
\begin{aligned}
& \text { elements of tempC; } \\
& \mathrm{Dj}=\mathrm{Dj} \cup\{\mathrm{Si}\} ; \quad \text { if } \quad \operatorname{tempC}(\mathrm{i}, \mathrm{j})=1, \quad \text { then } \\
&
\end{aligned}
$$

iii) Now $\operatorname{Di}(1 \leq i \leq n)$ is the place dependency set of the place Si. All the place dependency sets is iterated through, and confirm that all the place dependency sets contain the initial place. If the above condition is satisfied, the $\mathrm{xP} / \mathrm{T}$ system is reachable, otherwise it is unreachable.

2) Liveness verification

Since the $\mathrm{xP} / \mathrm{T}$ system has one and only one source place $i \in S$ and one sink place o $\in S$,which respectively satisfies $* \mathrm{i}=\phi$ and $\mathrm{o}^{*}=\phi$. If the reachability is met, it can be inferred that all the transitions are in the path from the source place to the sink place. Liveness versification can be illustrated from the following two aspects:

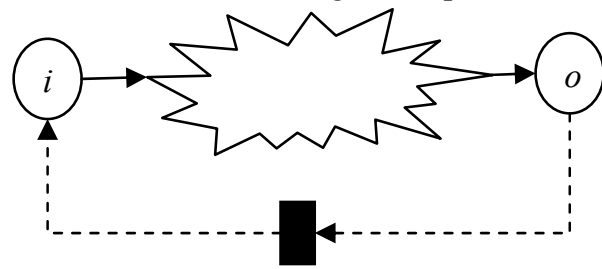

Figure 7. $\mathrm{xP} / \mathrm{T}$ System with the added transition

i) If a transition is located behind the place with tokens, i.e., the transition is closer to the sink place, then the transition may be enabled and it is in the path towards the sink place.

ii) If a transition is located in the front of the place with tokens, the state of the transition has two possibilities:

a) If the transition has fired, the transition is living.

b) If the transition is not fired due to the dissatisfaction with the firing condition of workflow case expressions, a transition can be added between the sink place and the source place, which will make the workflow case re-execute. So the liveness is not associated with the workflow case, but associated with the $\mathrm{XP} / \mathrm{T}$ system model.

\section{3) Integrity verification}


The integrity is mainly that all the cases which may occur in the transaction processing systems can be described. The integrity can be interpreted by the conditional routing, and $2 \mathrm{~N}$ cases can be described through $\mathrm{N}$ workflow variables (see section the routing structure for the $x P / T$ system workflow model).

\section{CONCLUSIONS}

After the $\mathrm{P} / \mathrm{T}$ system modeling theory is studied, the workflow property data are introduced into the $\mathrm{P} / \mathrm{T}$ system, and makes it valid that the weight of the arc of the $\mathrm{P} / \mathrm{T}$ system is zero to resolve the problems that modeling based on the Petri net is implemented complicatedly. On the above basis the $\mathrm{xP} / \mathrm{T}$ system is put forward, which makes it simpler to model and validate the correctness easily.

\section{REFERENCES}

[1] Xu Liang, Zhang Li, Fan Zhi-qiang. An Approach of Rea-1 Time Workflow Modeling Based on UML [J]. Journal o f Computer Research and Development, 2010, 47(7): 1184-1191.

[2] Lv Gang. The Research on Flexible Workflow Modeling Method [D]. Harbin: Harbin Engineering University,2012.

[3] HUANG Yu-yue, LI Ke-qing. Survey:legitimacy verification of workflow based on Petri net [J]. Application Research of Computers, 2013, 30(1): 26-29.
[4] ZHANG Min, GUO Yu-bin, LI Xi-ming, JIANG Yu. Survey for correctness problem of workflow system [J]. Application Research of Computers, 2009, 26(5): 1645-1647.

[5] WANG li-jun, GUO Xiao-dong. Approach to Verify Soundness of Workflow Process Models Based on Petri Net [J]. Journal of Jilin University ( Information Science Edition), 2011,29(6): 590-594.

[6] N R Adam, V Atluri, W K Huang. Modeling and Analysis of Workflows Using Petri Nets [J]. Journal of Intelligent Information Systems, 1998, (10): 131-158.

[7] T Basten. In Terms of Nets: Systems Design with Petri Nets and Process Algebra [D]. PhD thesis, Eindhoven University of Technology, Eindhoven, The Netherlands, 1998.

[8] W M P van der Aalst. Three good reasons for using a Petri net based workflow management system[C], Navathe and $T$ Wakayama, editors, Proceedings of the International Working Conference on Information and Process Integration in Enterprises (IPIC'96),Cambridge, Massachusetts, 1996: 179-201.

[9] LUO Jun zhou, SHEN Jun, GU Guan qun. From Petri Nets to Formal Description Techniques and Protocol Engineering [J], Journal of Software, 2000, 11(5): 606-615.

[10] Murata T. Petri Nets: Properties, Analysis and Applications[C] Proceedings of the IEEE, 1989, 77: 541-580.

[11] GAO De-ping, ZHOU Guo-xiang. Workflow model research and application based on colored Petri nets [J]. Journal of Hefei University of Technology, 2010, 33(2): 201-203

[12] LIU Lei. Research on workflow modeling method and its application based on Petri network[D]. Hangzhou: Zhejiang Scitech University,2013. 